\title{
PROSPECTS FOR CP VIOLATION MEASUREMENTS IN $\mathrm{LHCb}$
}
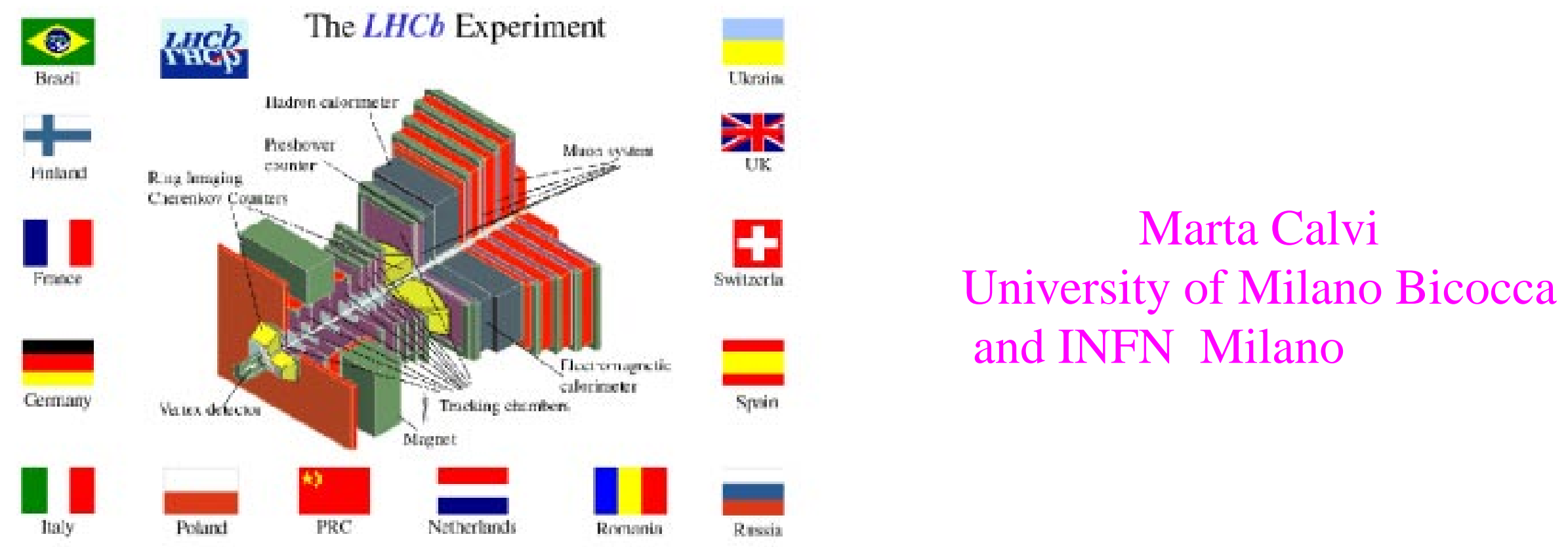


\section{At day 1 of LHCb (2005):}

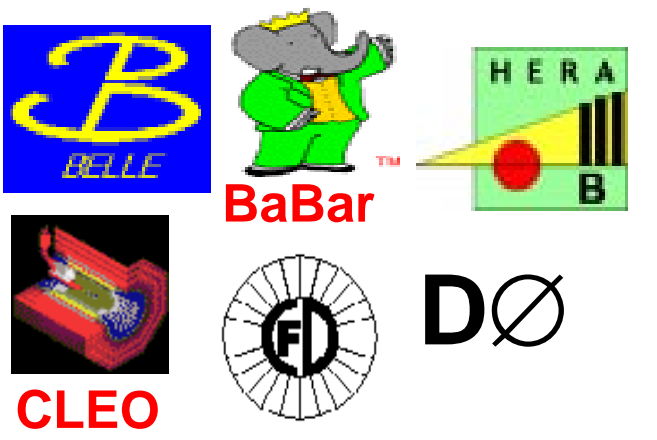

Will have provided:

- accurate measurements of $\left|\mathrm{V}_{\mathrm{cb}}\right|,\left|\mathrm{V}_{\mathrm{ub}}\right|,\left|\mathrm{V}_{\mathrm{td}}\right|$, rare $B$ decays channels, perhaps $\Delta \mathrm{m}_{\mathrm{S}}$

- $\sin (2 \beta)$ from $B_{d} \rightarrow J / \psi K_{s}$ with $\sigma \leq 0.05$

\section{Few examples:}

- $\mathrm{B}_{\mathrm{d}}^{0} \rightarrow \mathrm{J} / \psi \mathrm{K}_{\mathrm{s}}^{0}$ :

$$
A_{\mathrm{CP}}(\mathrm{t})=A_{\text {dir }} \cos (\Delta \mathrm{m} \mathrm{t})+A_{\text {mix }} \sin (\Delta \mathrm{m} \mathrm{t})
$$

high statistics is necessary to have sensitivity on $A_{\text {dir }}$

(LHCb 1 year's data)

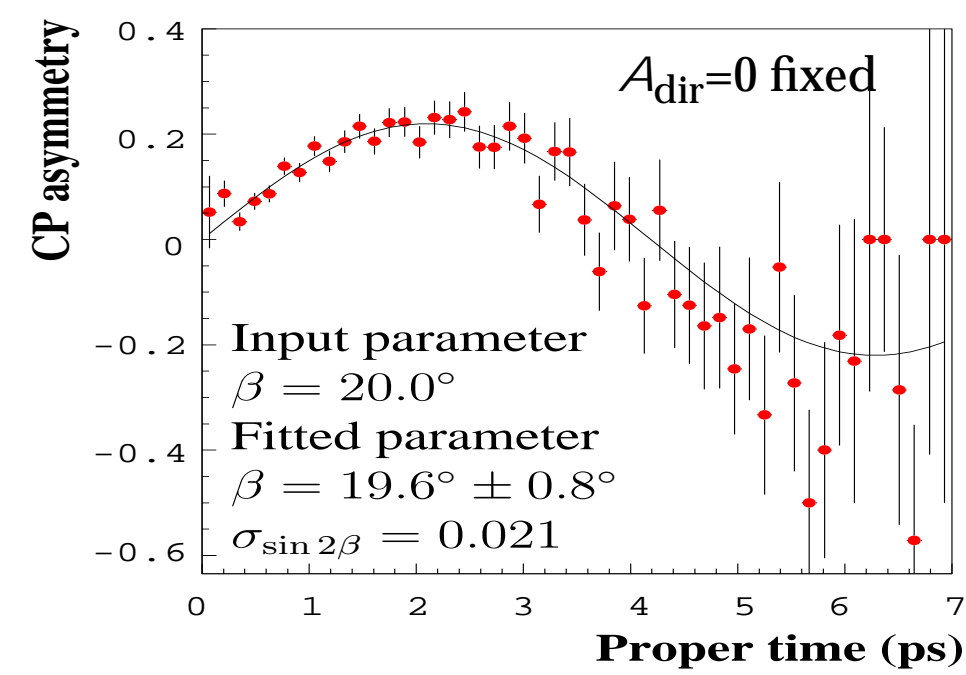


- New Physics

in $\mathrm{B}^{0} \overline{\mathrm{B}}^{0}$ mixing?

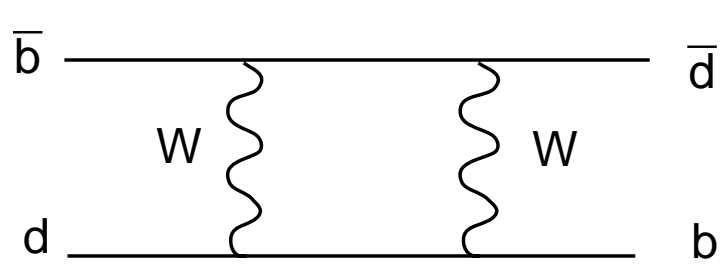

b

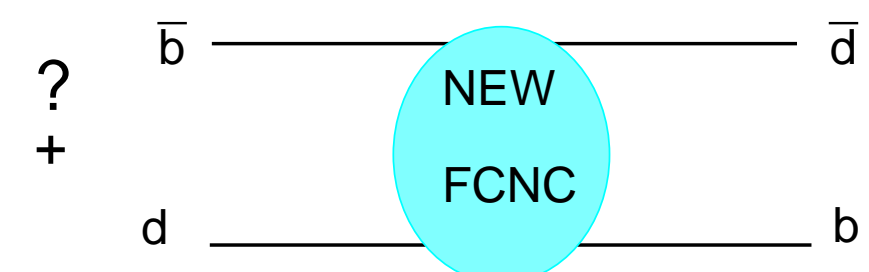

both $\mathrm{B}^{0}$ oscillations and $\mathrm{B}_{\mathrm{d}}^{0} \rightarrow \mathrm{J} / \psi \mathrm{K}^{0}$ asymmetry would be affected: $\beta$ measurements biased

From CP asymmetry in $\mathrm{B}_{\mathrm{d}}^{0} \rightarrow \mathrm{D}^{*}{ }^{-} \pi^{ \pm}:-2 \beta+\Phi_{\mathrm{NP}}-\gamma$

subtracting from

$$
\mathrm{B}_{\mathrm{d}}^{0} \rightarrow \mathrm{J} / \psi \mathrm{K}_{\mathrm{s}}^{0}:-2 \beta+\Phi_{\mathrm{NP}}
$$

\section{a clean determination of $\gamma$ and a test of New Physics}

\section{Experimental requirements:}

- High statistics on $B_{d}$ and $B_{s}$ to reach channels with small BR's $\left(<10^{-7}\right)$

- Measure rapid $B_{s}$ oscillations: good proper time resolution

- Good efficiency on several final states, including hadronic multi-body :

- sensitive trigger (leptonic and hadronic final states)

- particle identification

- good mass resolution 


\section{B production at $\mathrm{LHC}$}

pp collisions at $\sqrt{ } \mathrm{s}=14 \mathrm{TeV}:$

$$
\begin{aligned}
& \sigma_{\text {inel }} \approx 80 \mathrm{mb} \\
\sigma_{\mathrm{b} \overline{\mathbf{b}}} & \approx 500 \mu \mathrm{b} \quad \mathrm{S} / \mathrm{B} \sim 1 \%
\end{aligned}
$$

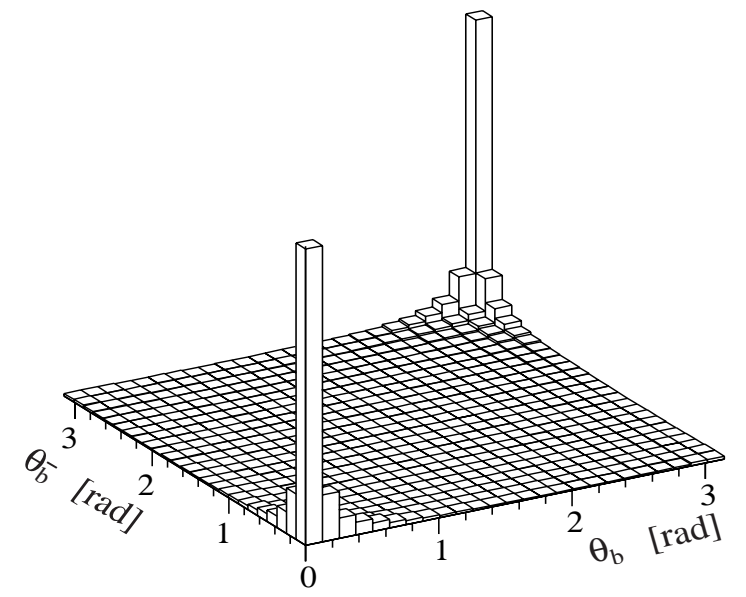

\section{Several pp interactions per bunch crossing}

LHCb chooses to work with single interaction

Advantages for: radiation damage detector occupancy triggering pattern recognition, flavour tagging

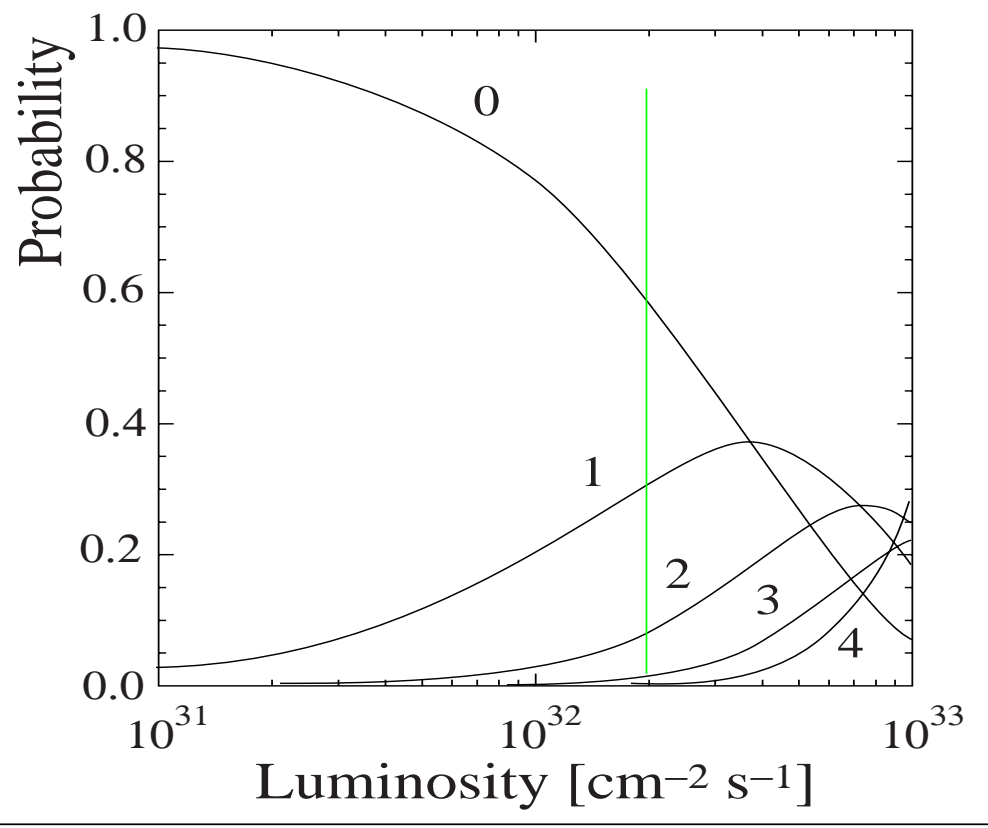


The detector will be operated at

beams defocussed at $\mathrm{LHCb}$ interaction point

D compatible with LHC running @1 $10^{34} \mathrm{~cm}^{-2} \mathrm{~s}^{-1}$

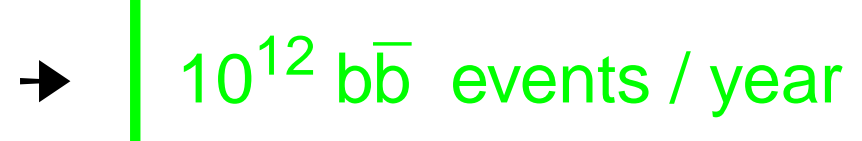

$\star$ Charged particle multiplicity

New tuning of simulation on UA5, CDF data after LHCb Tecnical Proposal:

(PYTHIA 6.1, multiple parton-parton interaction model, CTEQ4L structure function "post-HERA")
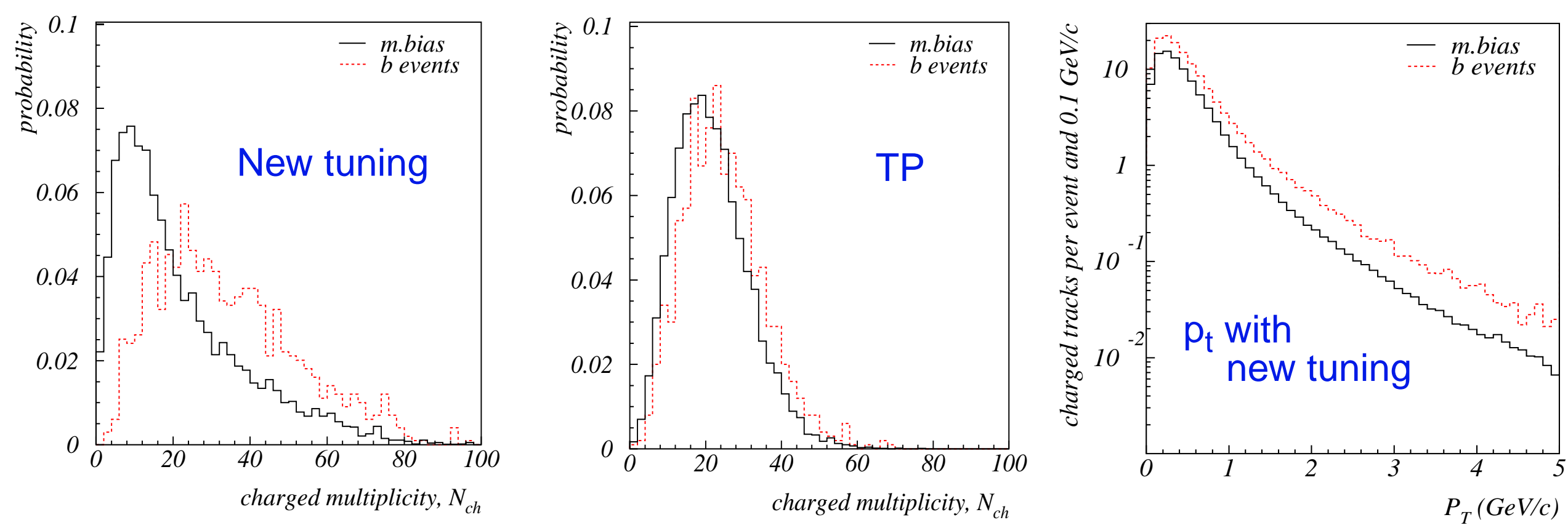

but TRIGGER performances are not affected! 
Bending Plane

Muon Detector

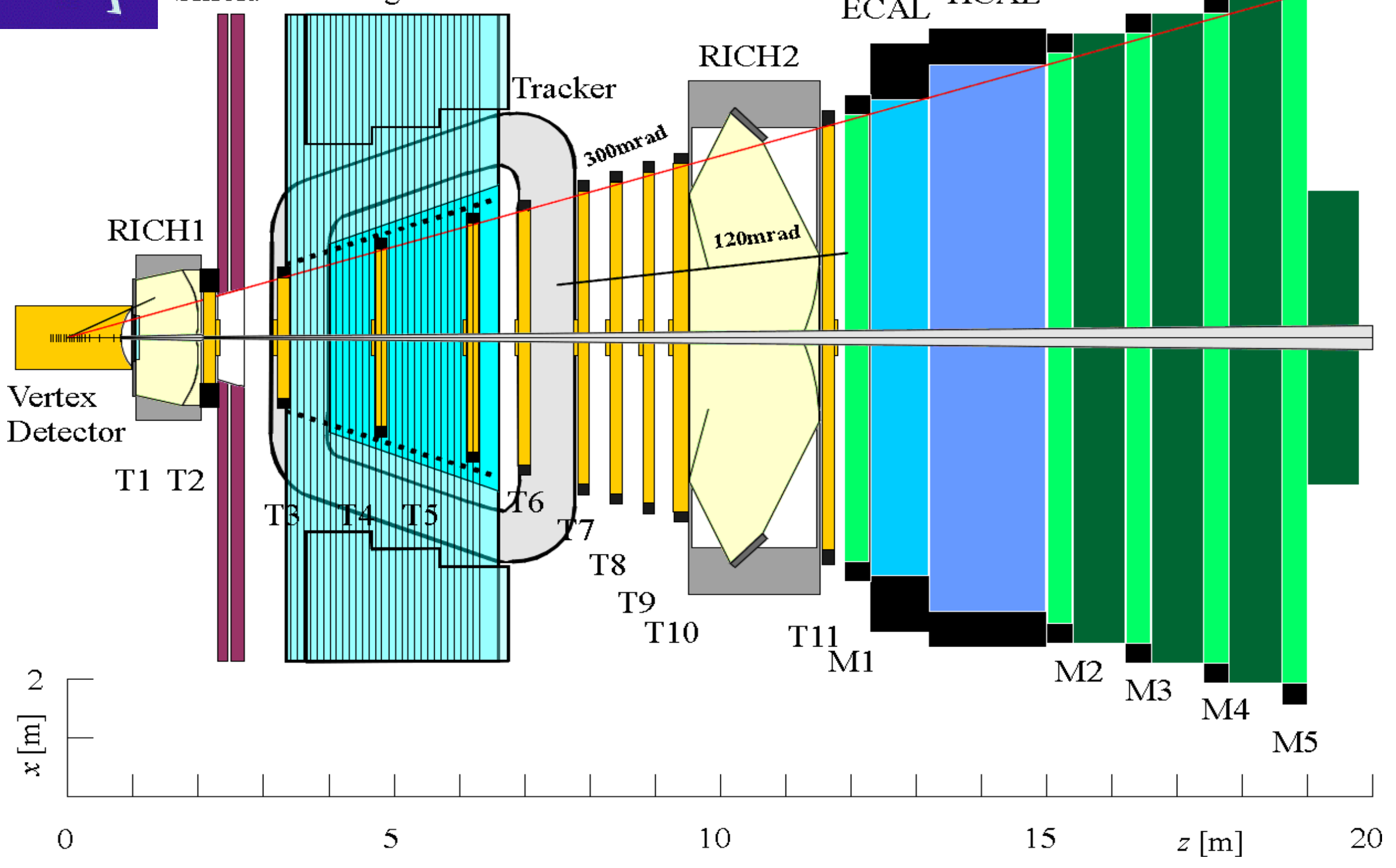




\section{$\mathrm{LHCb}$ Milestones}

$\square$ Feb $1996 \quad L H C b$ Letter of Intent

$\square$ Sep 1998 Approval of Technical Proposal

口 2000-2002 Technical Design Report of Detector Subsystems:

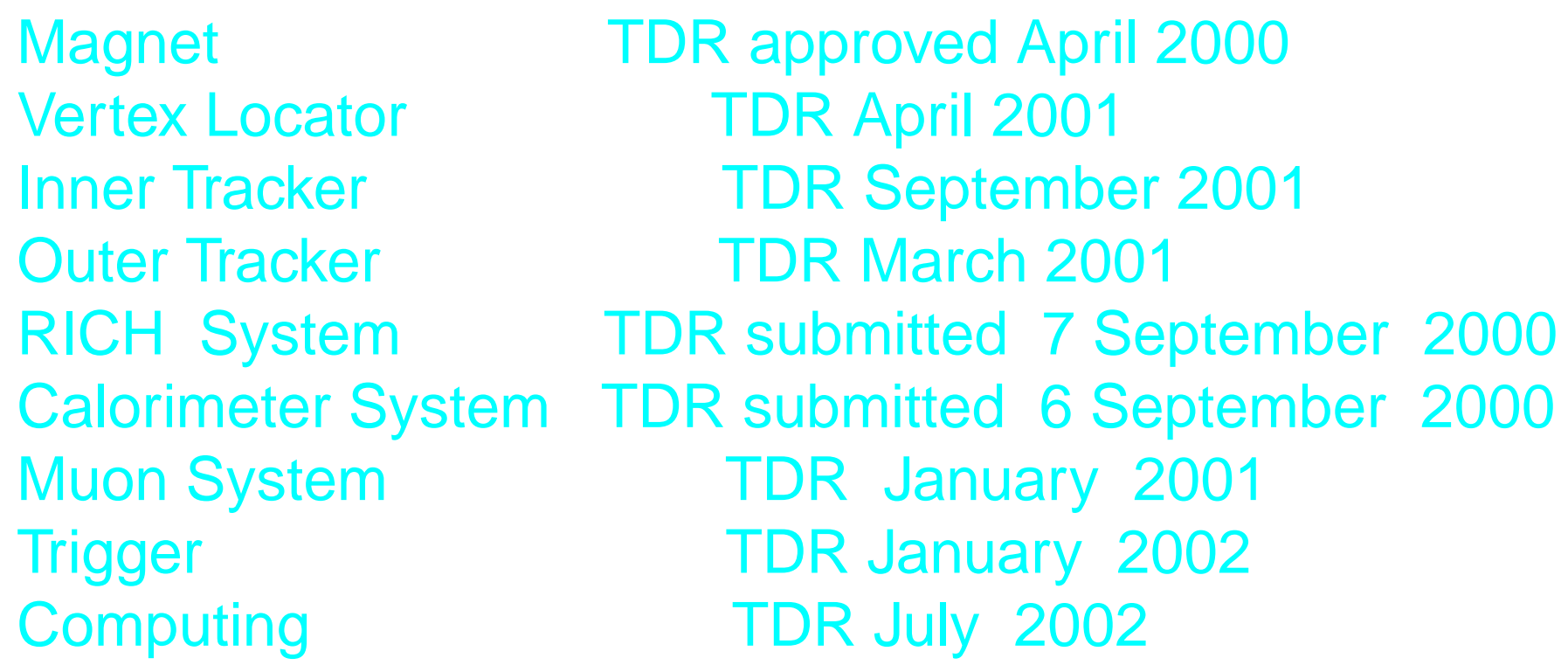

D 2000- July 2004 Construction Phase

$\square$ July 2005 1st beam in $\mathrm{LHCb}$ 


\section{GOALS:}

\section{LHCb TRIGGER}

$40 \mathrm{MHz}$ trigger, robust and flexible (not relying on a single subdetector) efficient on several B decay topologies (also fully hadronic final states)

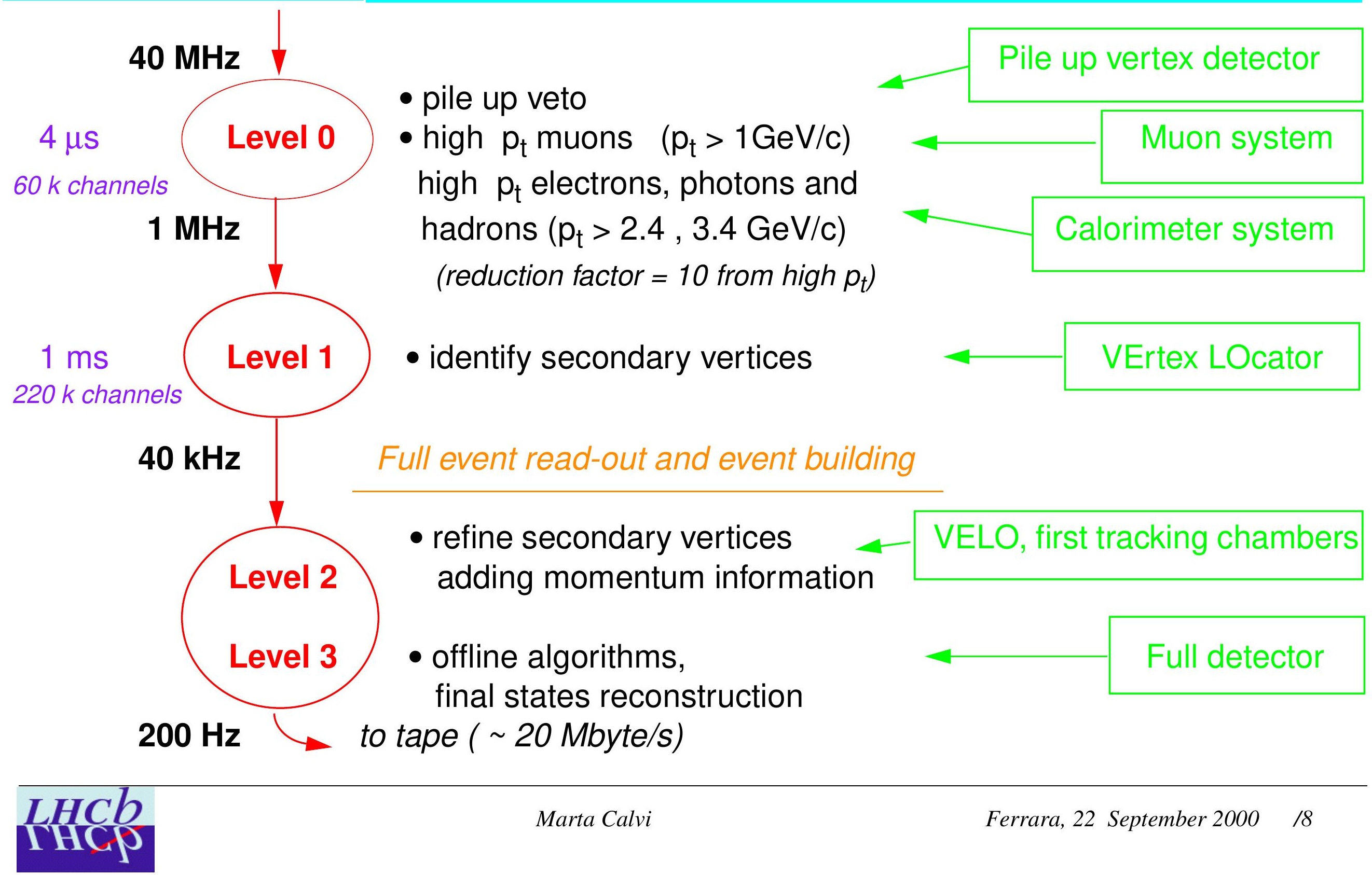




\section{TRIGGER Efficiency}

\begin{tabular}{|c|c|c|c|c|}
\hline & $(\%)$ & $L 1(\%)$ & $\mathrm{L} 2(\%)$ & Total(\%) \\
\hline $\mathrm{B}_{\mathrm{d}} \rightarrow \mathrm{J} / \psi(\mathrm{e}$ e $) \mathrm{K}_{\mathrm{s}}+$ tag & 72 & 42 & 81 & 24 \\
\hline $\mathrm{B}_{\mathrm{d}} \rightarrow \mathrm{J} / \psi(\mu \mu) \mathrm{K}_{\mathrm{s}}+$ tag & 88 & 50 & 81 & 36 \\
\hline $\mathrm{B}_{\mathrm{s}} \rightarrow \mathrm{D}_{\mathrm{s}} \mathrm{K}+\operatorname{tag}$ & 54 & 56 & 92 & 28 \\
\hline $\mathrm{B}_{\mathrm{d}} \rightarrow \pi \pi+$ tag & 76 & 48 & 83 & 30 \\
\hline $\mathrm{B}_{\mathrm{d}} \rightarrow \mathrm{D}_{\mathrm{s}}^{+} \mathrm{K}^{*} \mathrm{O}$ & 37 & 59 & 95 & 21 \\
\hline
\end{tabular}

for reconstructed and correctly tagged events

L1 vertex

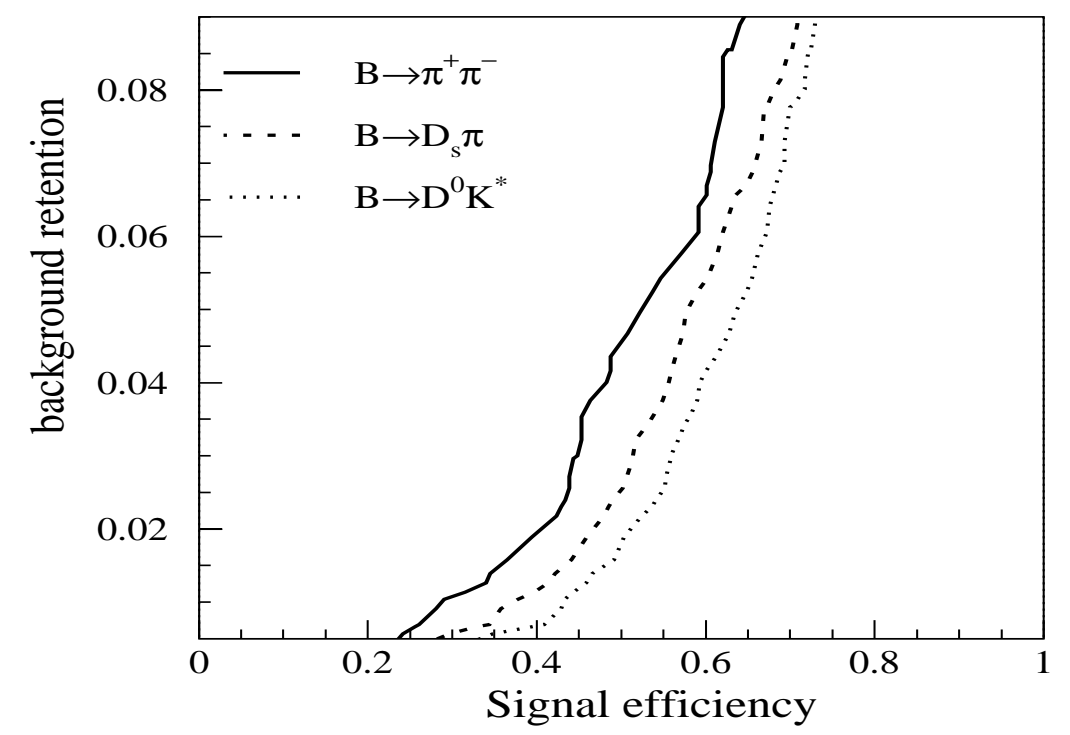

High $p_{t} \quad \mu$ trigger

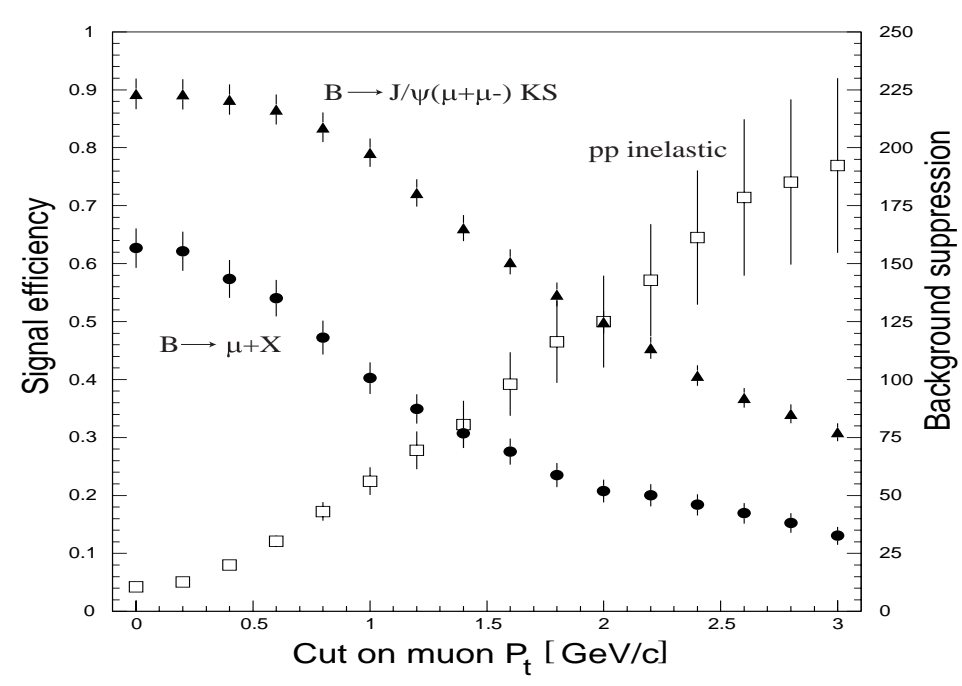

Future improvements of L1: will take into account LO informations to form a new decision

will increase efficiency, e.g. in $\mu \mu$ channels triggering on 2 high- $p_{t}$ muons 


\section{Vertex Locator (VELO)}
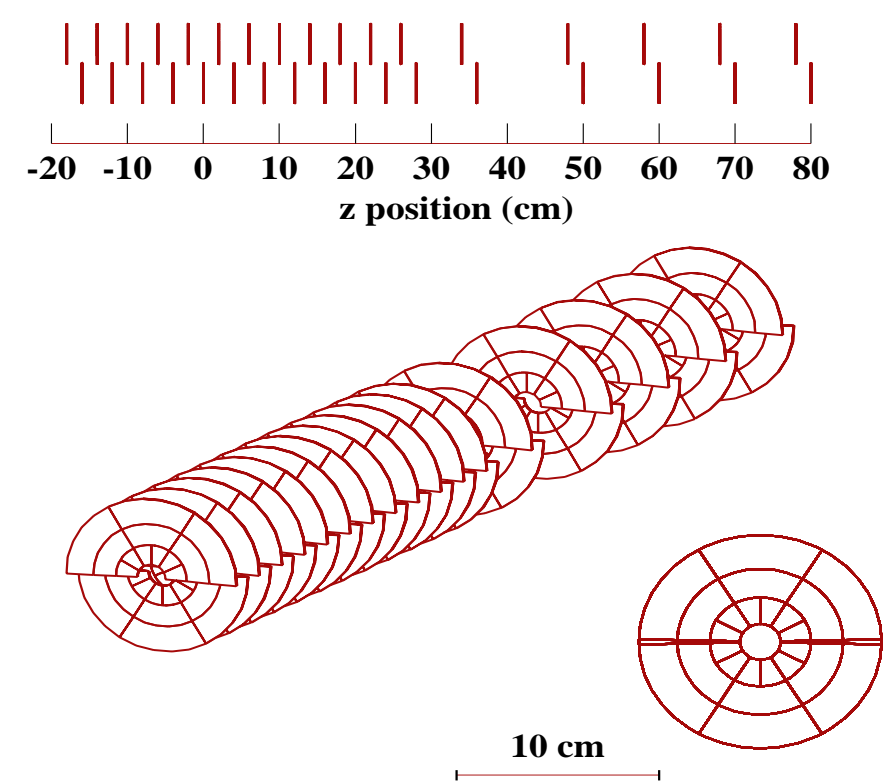

\section{Silicon strip detector}

in LHCb vacuum chamber

- 17 stations with $\mathrm{R}$ and $\phi$ measuring planes

- sensitive area $10 \mathrm{~mm}<r<60 \mathrm{~mm}$

- 2 half disks: retracted during injection by $3 \mathrm{~cm}$ to reduce radiation damage

\section{Optimization for TDR:}

\# of stations (25), positions, outer \& inner radii

- on average 9.5 hits / track

- Strips segmentation and pitch $(40 \mu \mathrm{m}-105 \mu \mathrm{m})$ determined by occupancy:

always below $1 \%$

- Number of readout channels $=2.2 \times 10^{5}$

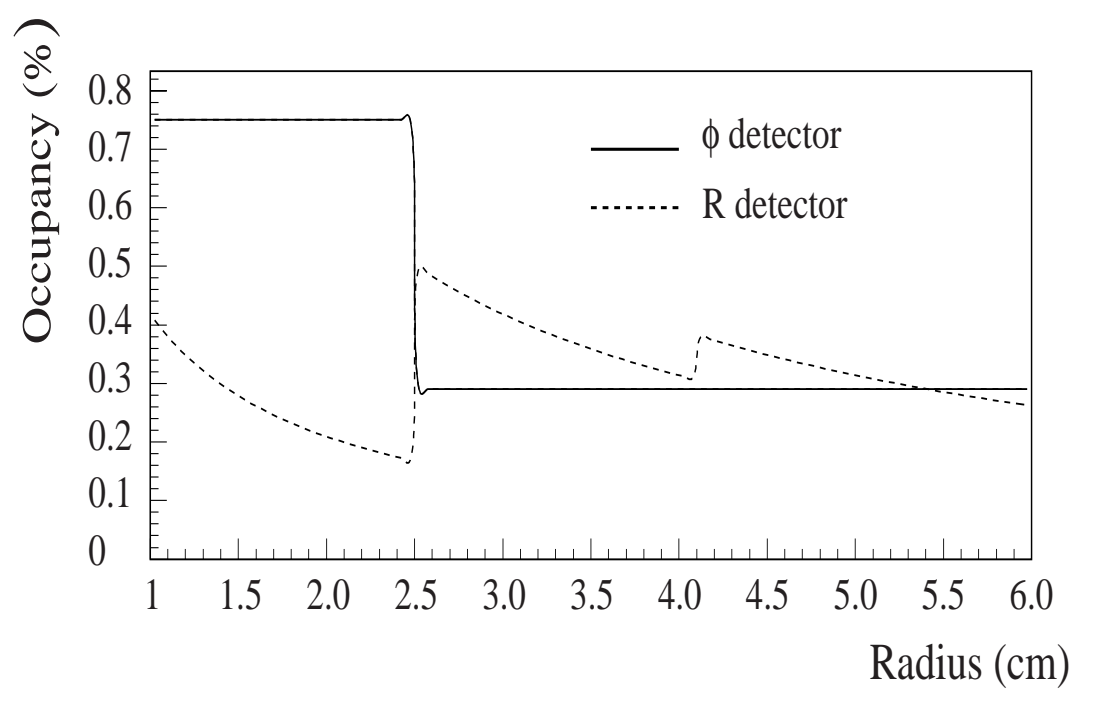




\section{Vertex Locator (VELO)}

- Single sided $200 \mu \mathrm{m} \mathrm{Si}$, double metal read out:

thin detector

S/B 12
- Impact parameter resolution:
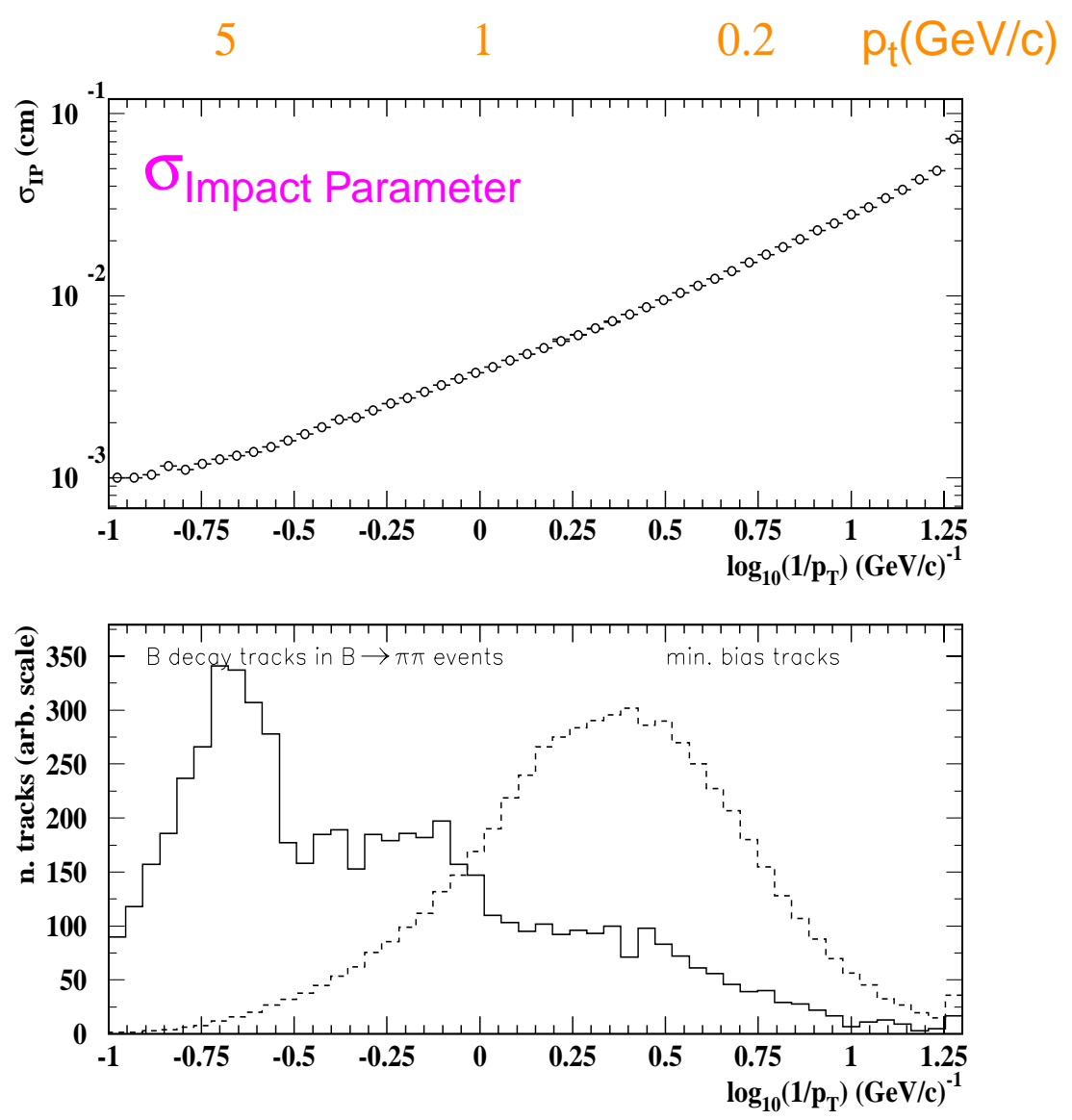

Accuracy on primary vertex:

$40 \mu \mathrm{m}$

- Front-end chip: 2 options under developement (DMILL SCTA128 or sub micron BEETLE )

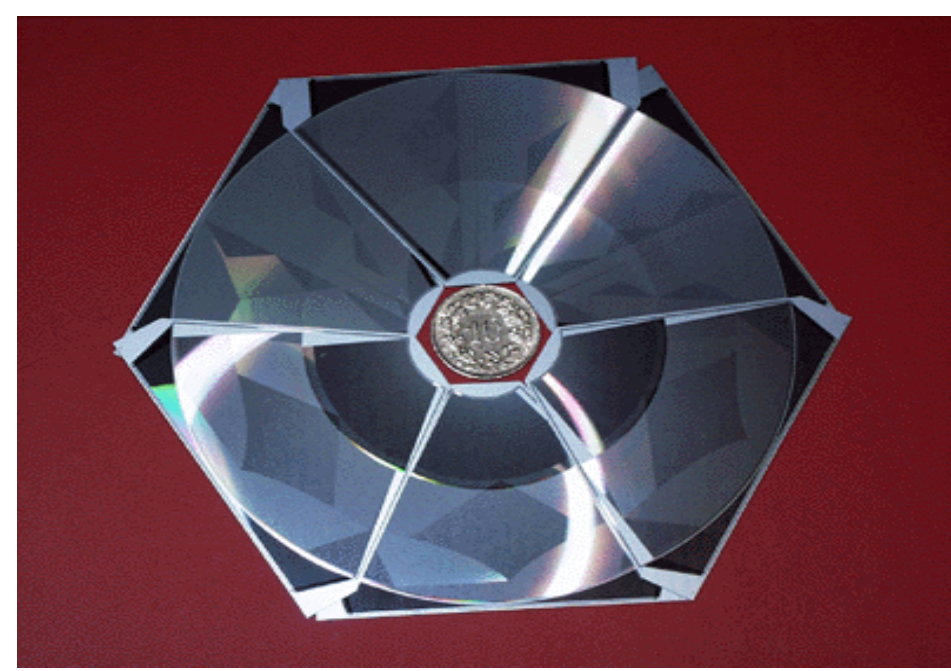

Prototype detector with $3 \mathrm{R}$ and $3 \phi$ sectors 


\section{Proper time resolution} for $B_{s} \rightarrow D_{s} \pi$

* time - dependent asymmetry measurements

* oscillations measurements

$$
\sigma_{\tau} \sim 43 \mathrm{fs}
$$

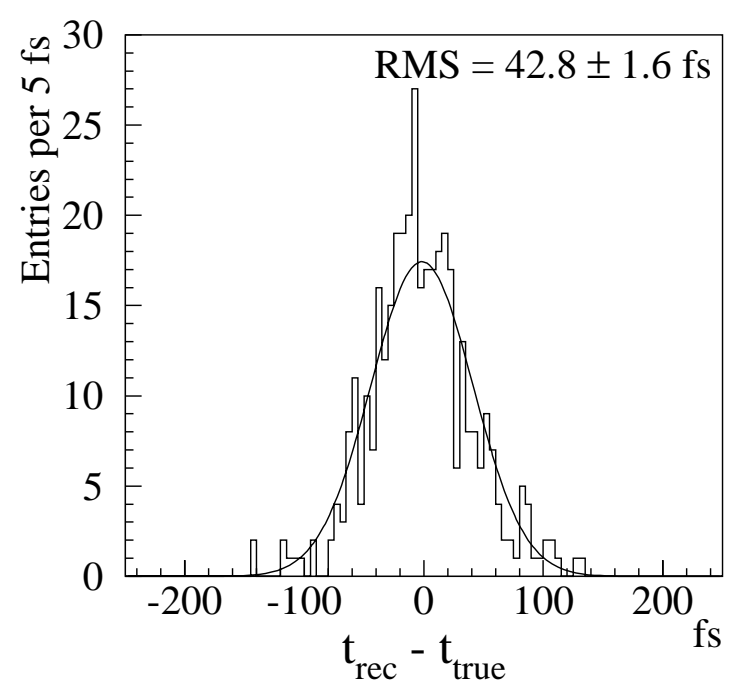

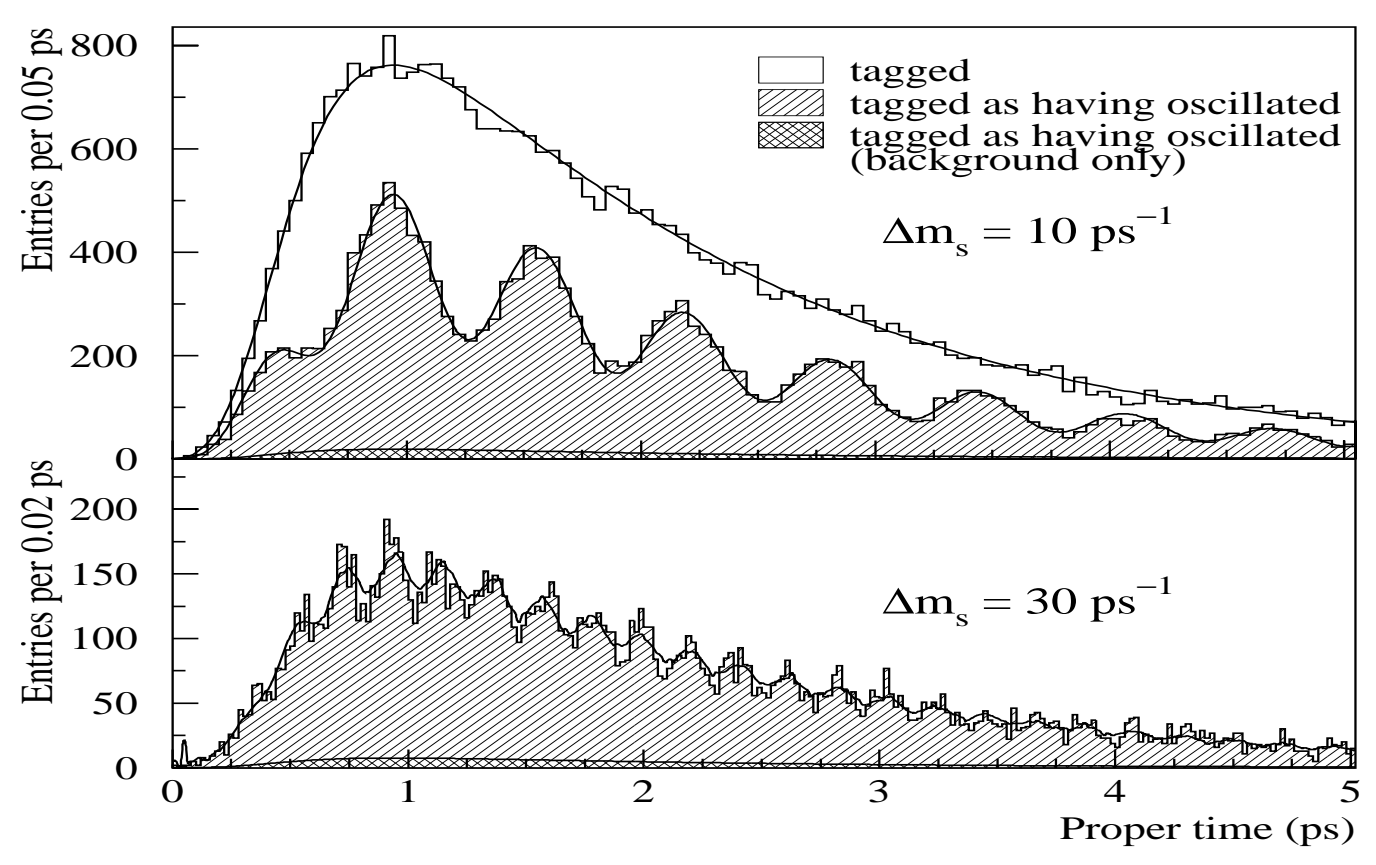

$>5 \sigma$ meaurement for $\Delta m_{s} \leq 48 p s^{-1}\left(x_{s}=75\right)$

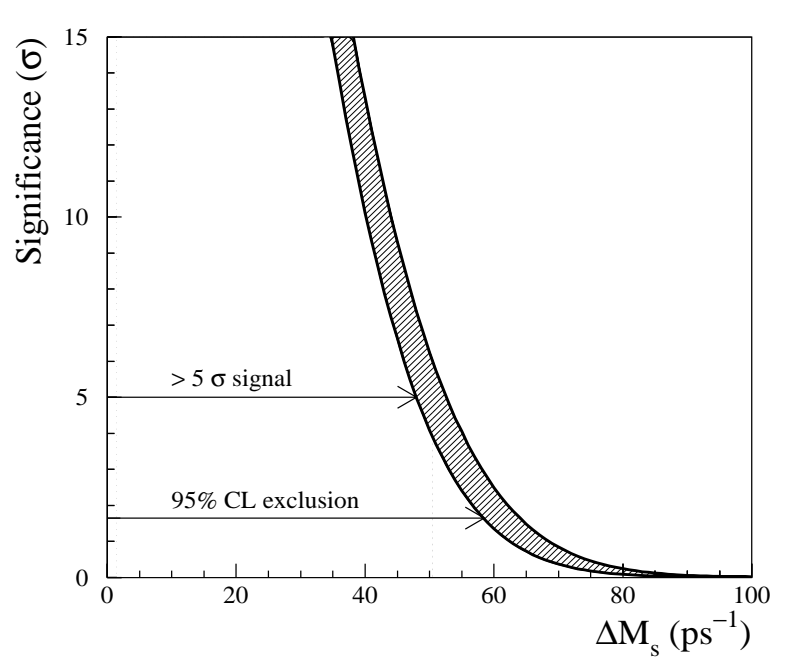




\section{MAGNET}
warm dipole
$4 \mathrm{Tm}$
(4.2 MW, yoke $1450 \mathrm{t}$ )

\section{TDR}

approved april 2000

\section{MAIN TRACKER}

OUTER TRACKER: straw drift tubes INNER TRACKER: triple GEM with 2D readout Si strips

$$
\begin{array}{r}
\sigma_{p} / p=0.3 \% \\
5 \mathrm{GeV} / \mathrm{c}<\mathrm{p}<200 \mathrm{GeV} / \mathrm{c}
\end{array}
$$

$$
\begin{array}{lll}
\sigma(M)=17 \mathrm{MeV} & \text { for } & \mathrm{B}_{\mathrm{d}} \rightarrow \pi^{+} \pi^{-} \\
\sigma(\mathrm{M})=4 \mathrm{MeV} & \text { for } & \mathrm{D}_{\mathrm{S}} \rightarrow \mathrm{K}^{-} \mathrm{K}^{+} \pi^{-}
\end{array}
$$




\section{RICH}

\section{$\mathrm{RICH} 1$}

Hadron identification in $\mathrm{LHCb}$ :

* Background suppression

$\Rightarrow$ high momentum hadrons in two-body $B$ decays

* B flavour tag identify $\mathrm{K}$ from $\mathrm{b} \rightarrow \mathrm{c} \rightarrow \mathrm{s}$ $\Rightarrow$ low momentum hadrons

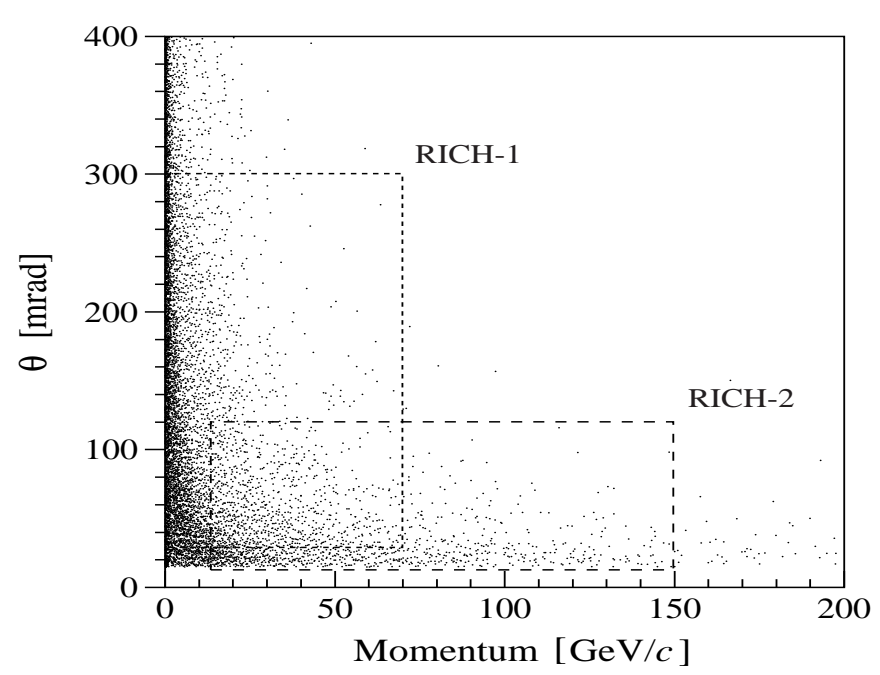

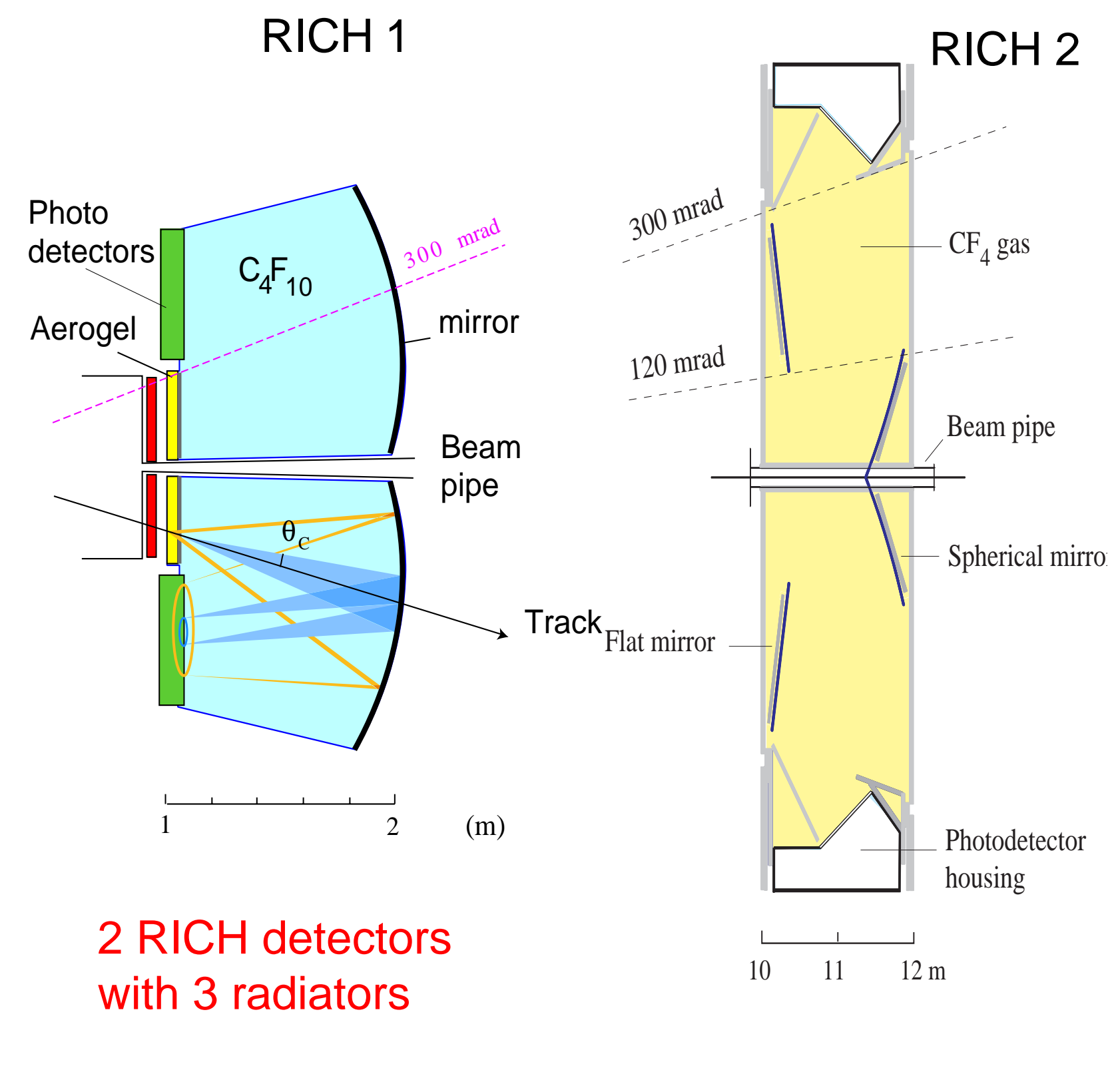




\begin{tabular}{l|ll|c} 
radiator & Aerogel & $\mathrm{C}_{4} \mathrm{~F}_{10}$ & $\mathrm{C} \mathrm{F}_{4}$ \\
lenght & $5 \mathrm{~cm}$ & $85 \mathrm{~cm}$ & $167 \mathrm{~cm}$ \\
$\quad \mathrm{n}$ & 1.03 & 1.0014 & 1.0005 \\
$\mathrm{p}_{\pi}$ thre. $(\mathrm{GeV} / \mathrm{c})$ & 0.6 & 2.6 & 4.4 \\
$\mathrm{p}_{\mathrm{K}}$ thre. $(\mathrm{GeV} / \mathrm{c})$ & 2.0 & 9.3 & 15.6 \\
$\sigma(\theta)(\mathrm{mrad})$ & 2.0 & 1.45 & 0.58 \\
$\mathrm{~N}$ pe & 6.6 & 32.7 & 22.5 \\
\hline
\end{tabular}

more than $3 \sigma \pi / \mathrm{K}$ separation in $3<\mathrm{p}<100 \mathrm{GeV} / \mathrm{c}$

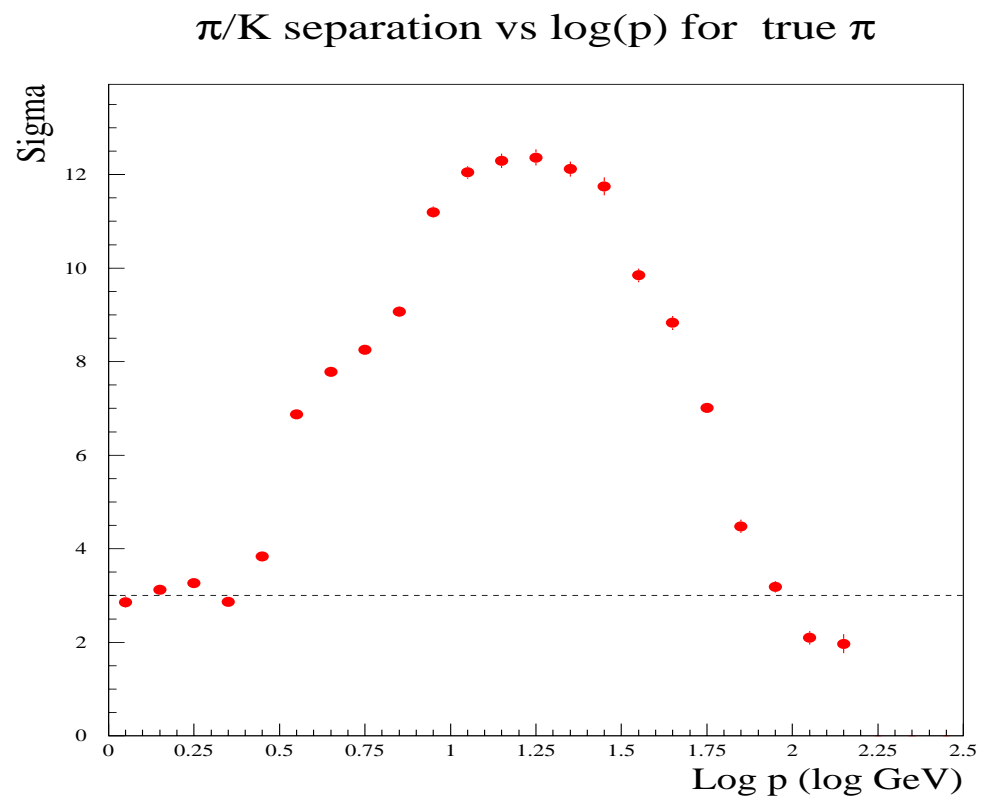

$\mathbf{B}_{\mathbf{d}} \rightarrow \pi \pi$
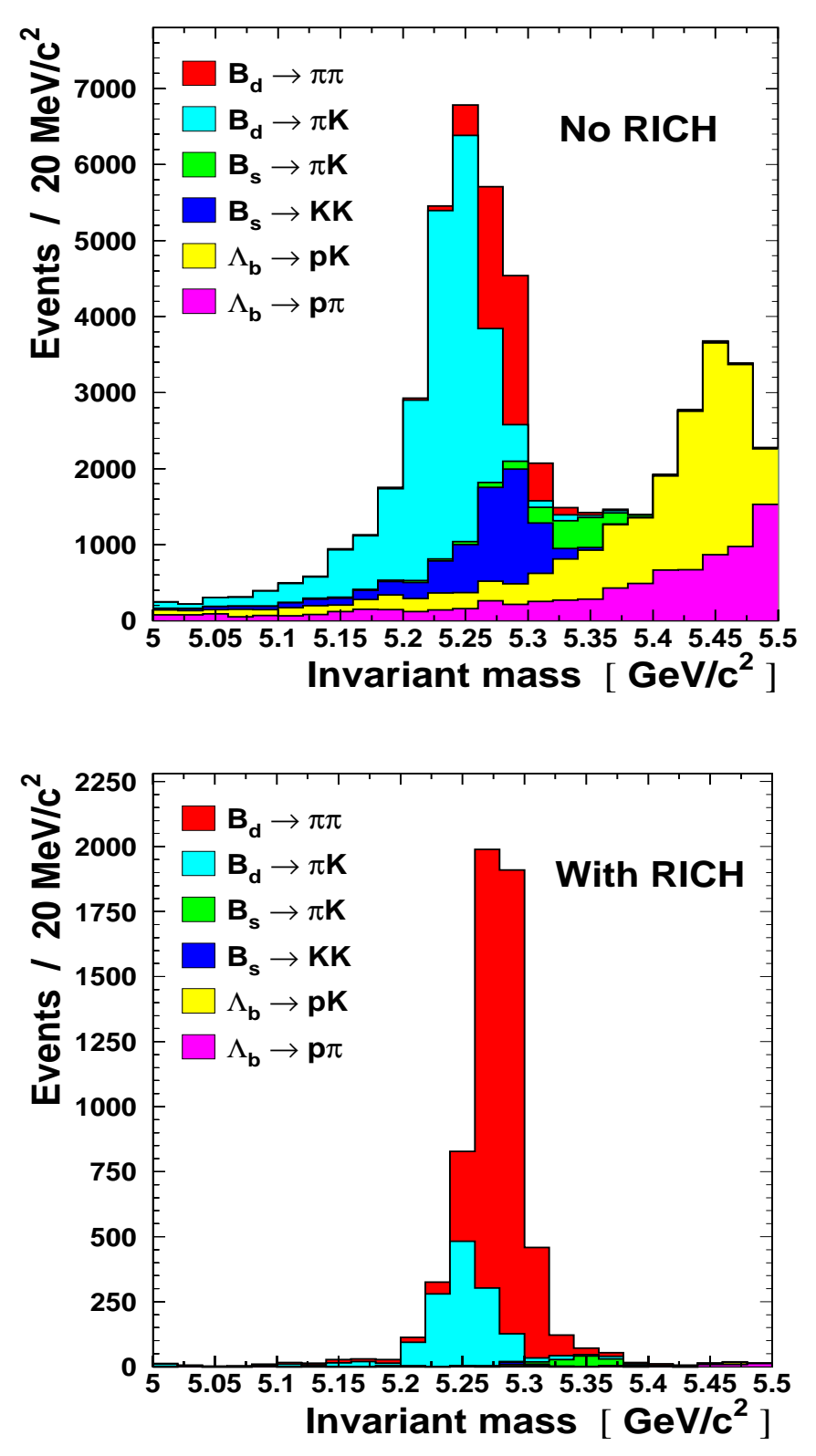


\section{RICH PHOTODETECTORS}

Requirements: $~ 3 \mathrm{~m}^{2}$ area, $2.5 \times 2.5 \mathrm{~mm}^{2}$ granularity, single photon sensitivity, high Q.E.

3 options considered:

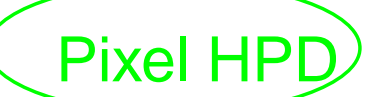

Pad HPD

$\downarrow$ base line solution

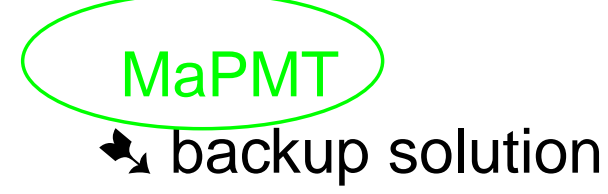

Pixel Hybrid PhotoDiode [ CERN and DEP (The Nederlands) project ]

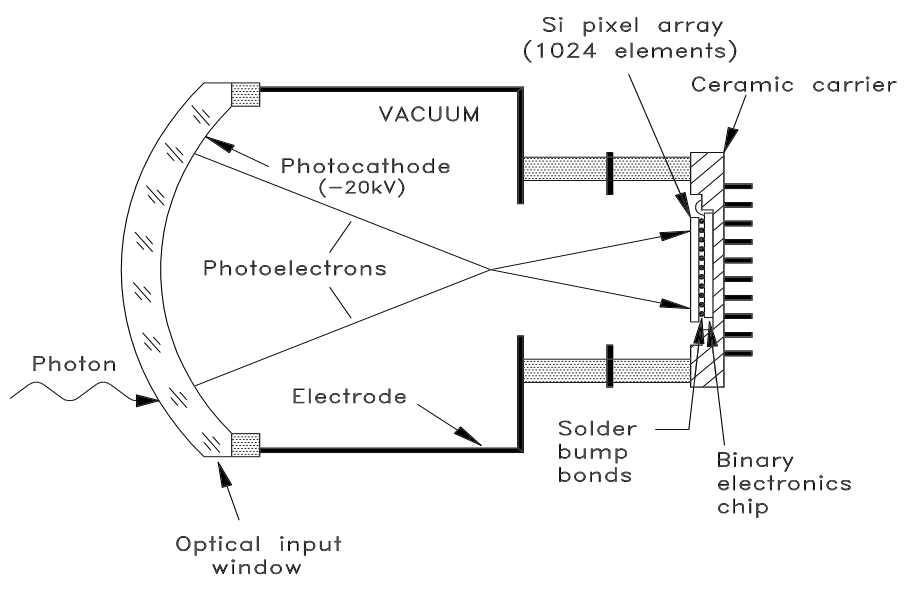

$\square 1024(500 \mu \mathrm{m} \times 500 \mu \mathrm{m})$ Si pixel sensor bump bonded to binary read out electronics - ALICE chip

$\square \int$ Q.E. $\mathrm{d} E \sim 0.77 \mathrm{eV}$

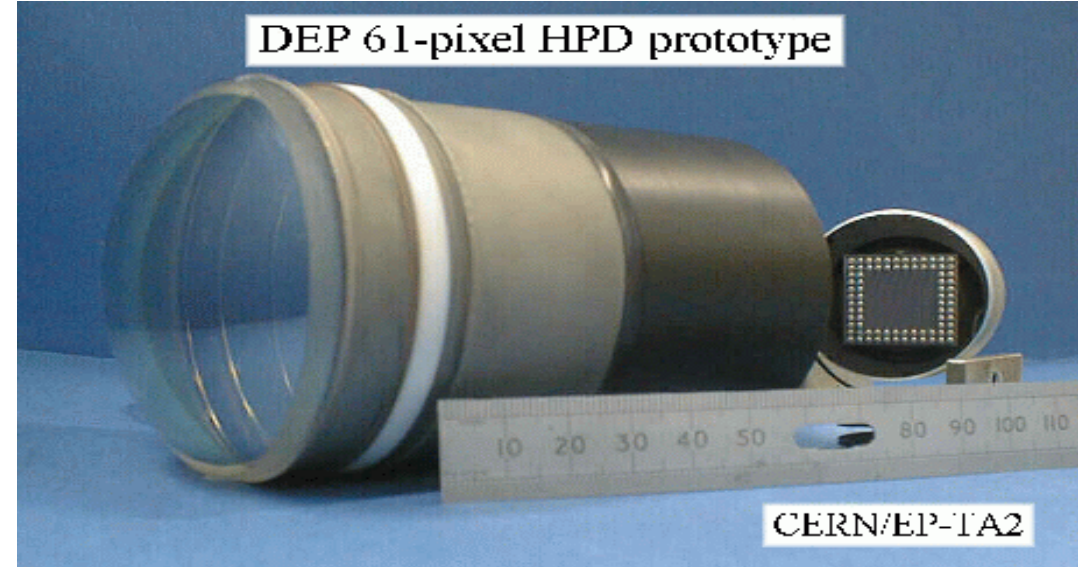

$\square 80 \mathrm{~mm}$ diameter envelope cross-focusing electrodes

$\square \quad 70 \%$ active area coverage ( 450 tubes, $\sim 325 \mathrm{k}$ channels) 


\section{TEST BEAM RESULTS}
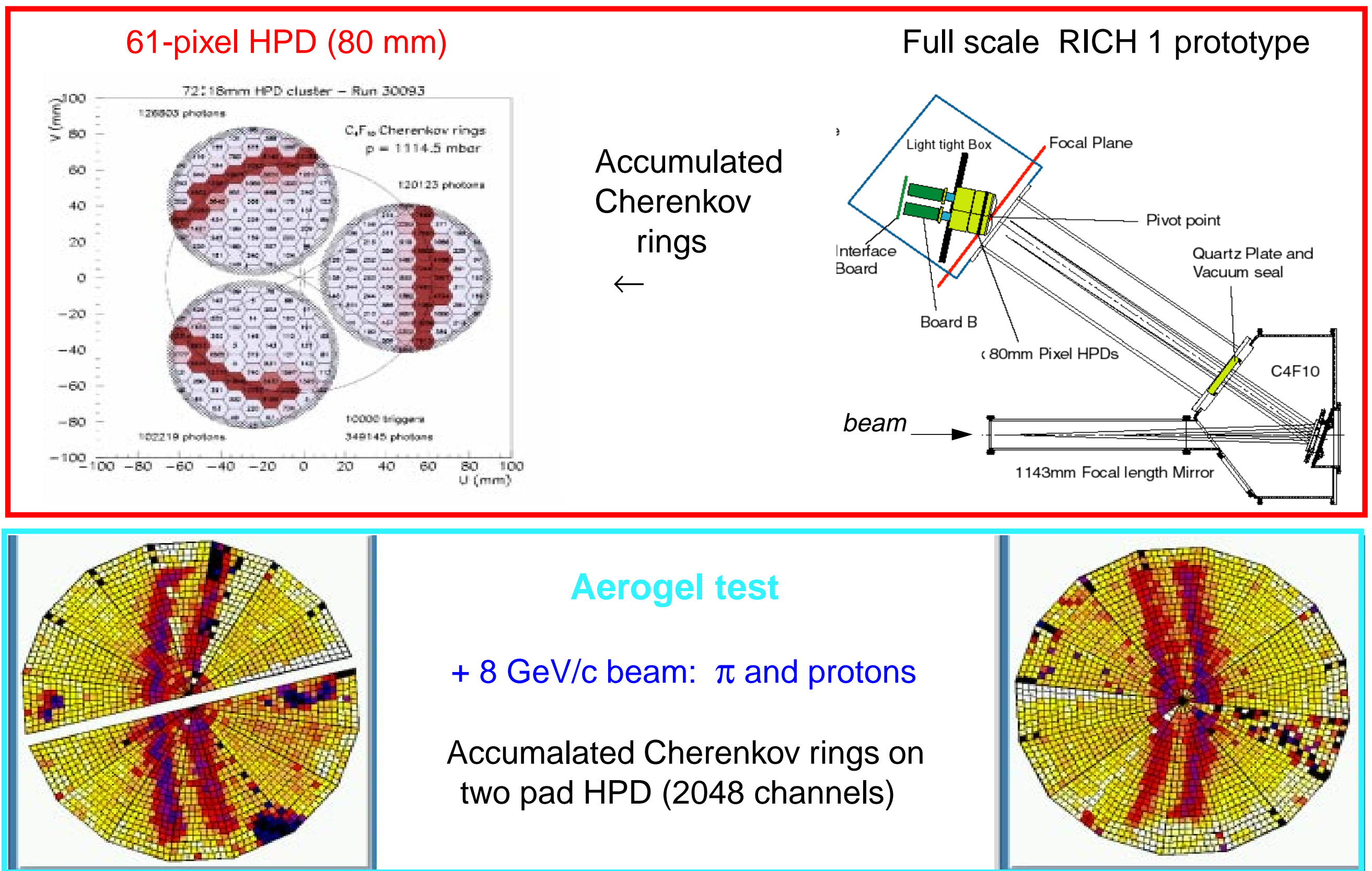

\section{Aerogel test}

$+8 \mathrm{GeV} / \mathrm{c}$ beam: $\pi$ and protons

Accumalated Cherenkov rings on two pad HPD (2048 channels)

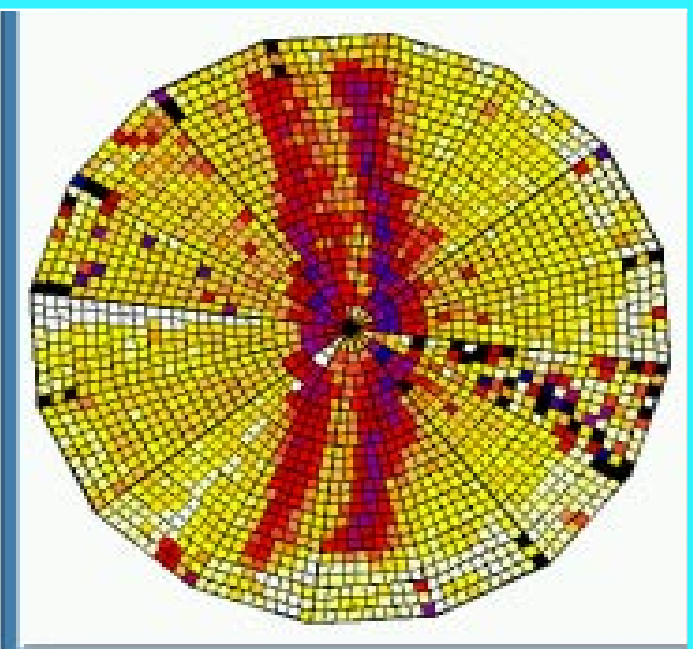




\section{CALORIMETERS}

L0 trigger requires: fast detector with longitudinal segmentation for e/ $\pi$ separation (electrons up to $200 \mathrm{GeV}$, hadrons up to $300 \mathrm{GeV}$ )

Hit density varies 2 order of magnitude over calorimeter surface:

variable lateral segmentation ( 3 zones in ECAL, 2 zones in $\mathrm{HCAL}$ )

Joint Calorimeter Test

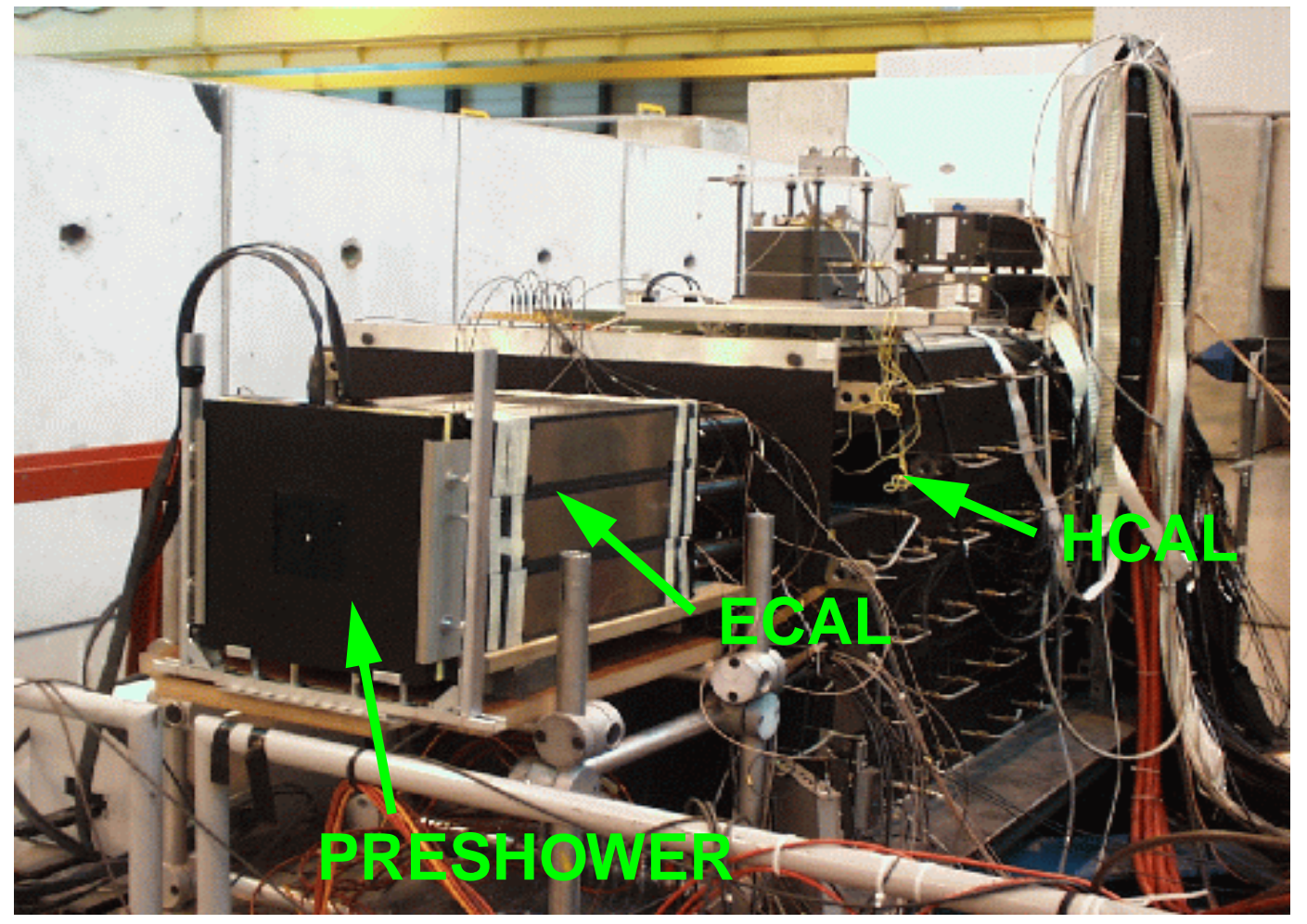

\section{Pre-shower: sandwich} scintillator-Pb-scintillator $2 \mathrm{X}_{0}$

ECAL: Shashlik type $25 \mathrm{X}_{0}$

$$
\sigma(E) / E=10 \% / \sqrt{ } E \oplus 1.5 \%
$$

HCAL: $\mathrm{Fe}+$ scintillating tiles, $5.6 \lambda_{1}$

$$
\sigma(E) / E=80 \% / \sqrt{ } E \oplus 10 \%
$$




\section{MUON CHAMBERS}

5 muon stations: $\quad 870 \mathrm{~m}^{2}$ of detector area,

$\sim 150 \mathrm{k}$ physical channels, $26 \mathrm{k}$ logical channels

Regions closer to the beam have stronger requirements for rate capability

$\square$ Rate $\leq 1 \mathrm{kHz} / \mathrm{cm}^{2} \quad(42 \%$ of Muon System)
$\mathrm{RPC}$ with 2 gaps (double gap or 2 single gap ORed)
Test beam results: $\quad \begin{aligned} & \text { time resolution of } 1.3 \mathrm{~ns} \text {, with efficiency }>99 \% \text { in } 10 \mathrm{~ns} \\ & \text { efficiency }>95 \% \text { at } 1.8 \mathrm{kHz} / \mathrm{cm}^{2}\end{aligned}$

$\square 1 \mathrm{kHz} / \mathrm{cm}^{2}<$ Rate $\leq 100 \mathrm{kHz} / \mathrm{cm}^{2} \quad(58 \%$ of Muon System $)$
MWPC with anode wire and/or cathode pad readout
good ageing properties when operated with $\mathrm{Ar} / \mathrm{CO}_{2} / \mathrm{CF}_{4} \quad 40 / 50 / 10$ mixture
Test beam results: $\quad \begin{aligned} & \text { time resolution }<3 \mathrm{~ns}, \text { with efficiency }>99 \% \text { in } 20 \mathrm{~ns} \\ & \text { good performances at rates }>100 \mathrm{kHz} / \mathrm{cm}^{2}\end{aligned}$
$\square$ Rate $>100 \mathrm{kHz} / \mathrm{cm}^{2} \quad(<1 \%$ of Muon System $)$
??? still under study

LHCb $\quad$ Marta Calvi $\quad$ Ferrara, 22 September $2000 \quad / 19$




\section{$\gamma$ from $B_{d} \rightarrow D^{* \overline{+}} \pi^{ \pm}$}

- CP asymmetries from interference between $\mathrm{B}_{\mathrm{d}} \rightarrow \mathrm{D}^{*-} \pi^{+}$and $\mathrm{B}_{\mathrm{d}} \rightarrow \overline{\mathrm{B}}_{\mathrm{d}} \rightarrow \mathrm{D}^{*-} \pi^{+}$decays

- Tiny effect $(\sim 1 \%)$ due to double Cabibbo suppressed $b \rightarrow u$ transition

- Extract $2 \beta+\gamma$ from time dependent fit of CP asymmetries. No penguin contributions.

exclusive reconstruction

$\sim 84 \mathrm{k} \mathrm{ev} /$ year S/B 12 (reconstructed and tagged)

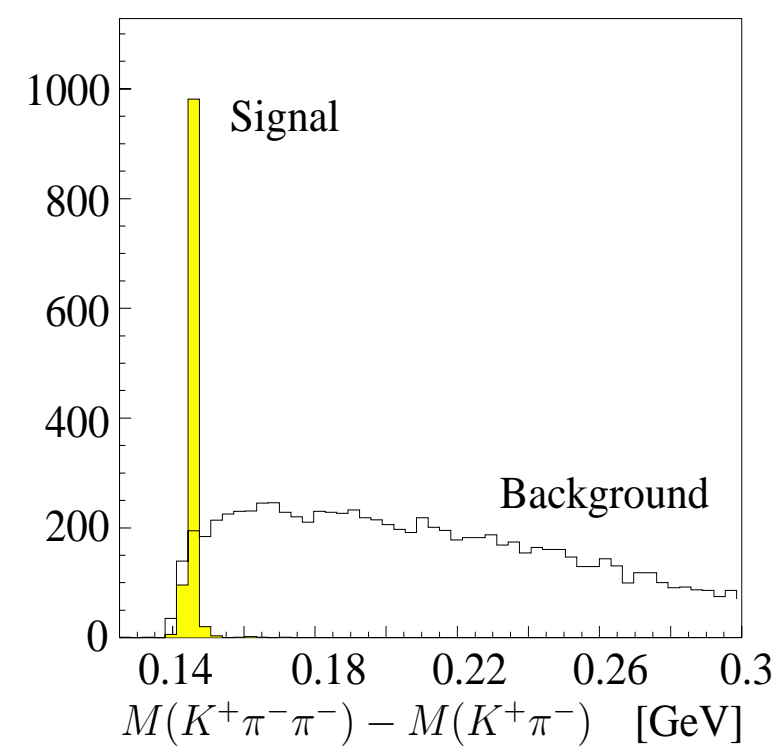

partial reconstruction

$\sim 260 \mathrm{k} \mathrm{ev} /$ year $\mathrm{S} / \mathrm{B} \sim 3$

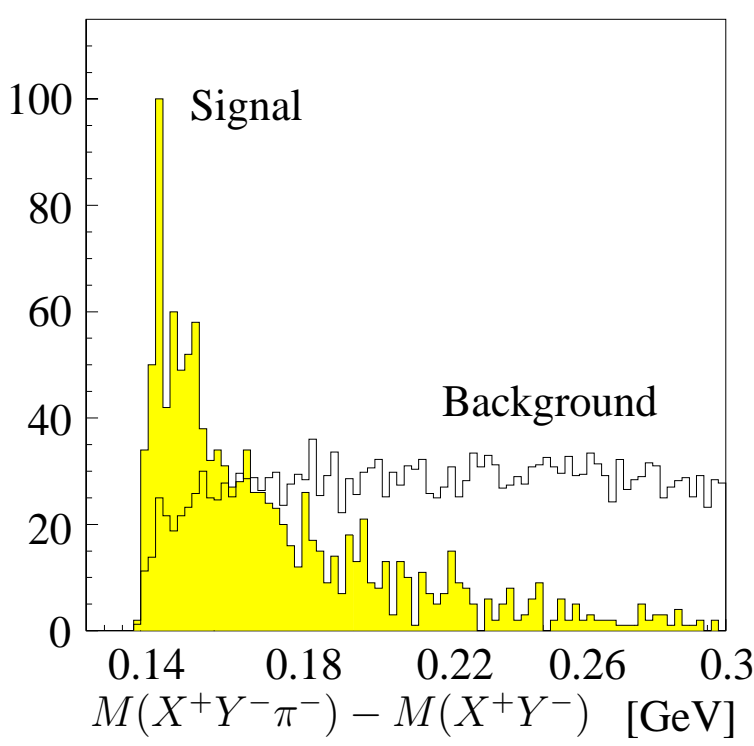

Sensitivity will depend on strong phase difference

$\mathrm{B}_{\mathrm{d}} \rightarrow \mathrm{D}^{{ }^{*}+} \mathrm{a}_{1}{ }^{ \pm}$can be added $\sim 360 \mathrm{k}$ events /year, S/B $\sim 4$. 


\section{$\gamma$ from $\mathbf{B}^{0}{ }_{\mathrm{s}} \rightarrow \mathbf{D}_{\mathrm{s}}^{\overline{+}} \mathbf{K}^{ \pm}$}

$B_{s}$ counterpart of $B_{d} \rightarrow D^{*-} \pi^{+}$but interference effects much larger. No penguins contributions. Extract $\gamma-2 \delta \gamma$ from time dependent fit of CP asymmetries. Assuming $2 \delta \gamma$ known from $\mathrm{B}_{\mathrm{S}} \rightarrow \mathrm{J} / \psi \phi$ this will be a clean measurement of $\gamma$

D Background from $\mathrm{B}_{\mathrm{s}}^{0} \rightarrow \mathrm{D}_{\mathrm{s}}{ }^{-} \pi^{+} \sim 20$ times higher --> RICH for $\mathrm{K} / \pi$ separation ( $\pi$ misid $0.1 \%$ ) D Fast $B_{s}$ oscillations --> good proper time resolution (VELO $\sigma(\tau)=43$ fs )
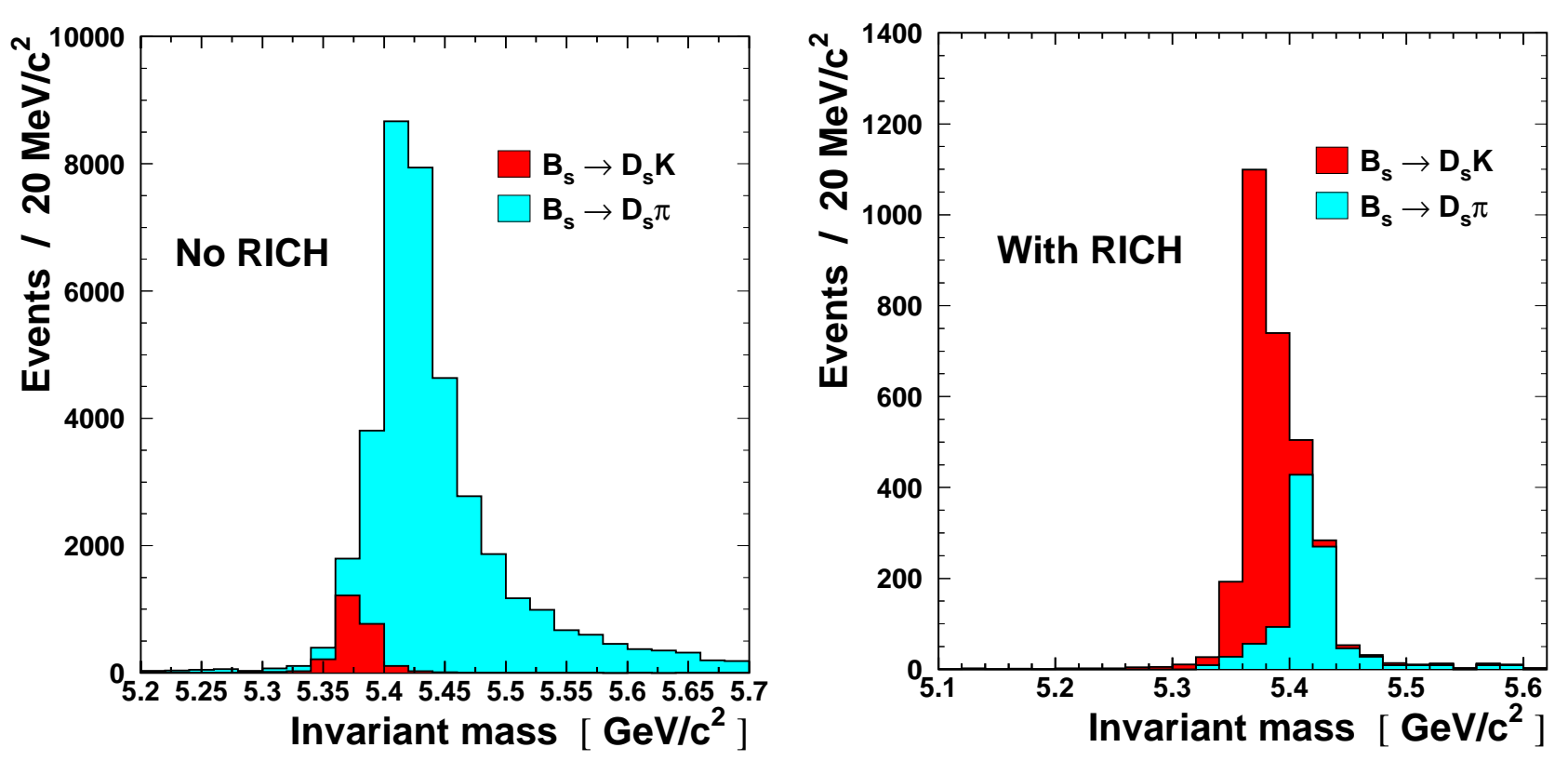

\section{0 events expected in 1 year (reconstructed and tagged) \\ reconstr. efficiency $0.5 \%$}

$$
\begin{gathered}
\sigma\left(\mathrm{M}_{\mathrm{B}}\right) \sim 11 \mathrm{MeV} \\
\mathrm{S} / \mathrm{B} \sim 12.5
\end{gathered}
$$

Sensitivity to $\gamma-2 \delta \gamma$ will depend on $\Delta \mathrm{m}_{\mathrm{S}}, \Delta \Gamma_{\mathrm{S}} / \Gamma_{\mathrm{S}}$, strong phase difference:

expected precision $\gamma-2 \delta \gamma: 6^{0}-12^{0}\left(\Delta \mathrm{m}_{\mathrm{S}}=15 \mathrm{ps}^{-1}\right)$ 


\section{$\alpha \quad$ from $B^{0}{ }_{d} \rightarrow \rho \pi$}

$$
\mathrm{B}_{\mathrm{d}}^{0} \rightarrow \underset{\rho^{0} \pi^{0}}{\rho^{-} \pi^{+} \rightarrow \pi^{+} \pi^{-} \pi^{0}}
$$$$
\sigma\left(\mathrm{M}_{\mathrm{B}}\right)=42 \mathrm{MeV}
$$

( $35 \mathrm{MeV}$ with $\pi^{0}$ constraint)

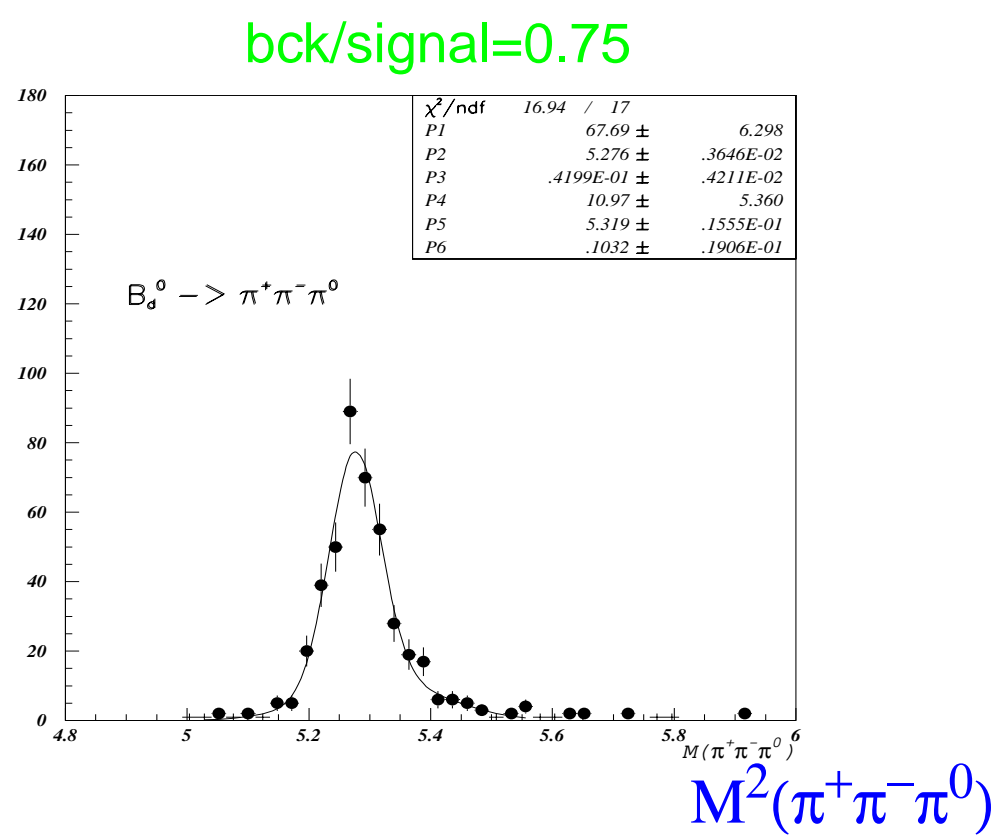

Tree and Penguins parameters, including $\alpha$, can be extracted from a full 3 body analysis, taking into account interference effects between vector mesons of different charge
Time dependent analysis of event distribution in Dalitz plot

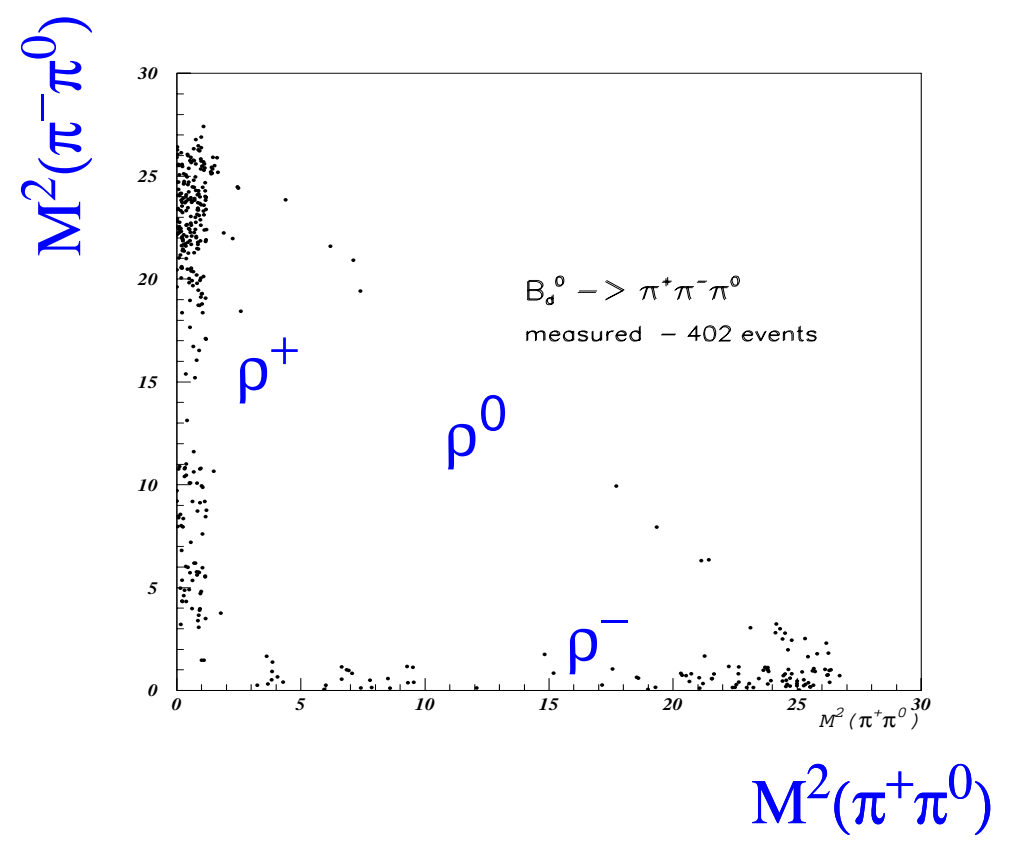

expected events: $\sim 1200 \rho^{ \pm} \pi^{\overline{+}}$

$$
\sim 100 \rho^{0} \pi^{0}
$$

Expected sensitivity: $\sigma(\alpha) \sim 3^{0}-6^{0}$ in 1 year 
Expected CP sensitivities in 1 year $\mathbf{L H C b}\left(10^{7} \mathrm{~s}, \mathrm{~L}=2 \times 10^{32} \mathrm{~cm}^{-2} \mathrm{~s}^{-1}\right)$

\begin{tabular}{|c|c|c|c|}
\hline Decay channel & $\begin{array}{c}\mathrm{N} \text { events } \\
\text { (reconstructed } \\
\text { and tagged) }\end{array}$ & parameter & sensitivity \\
\hline $\mathrm{B}_{\mathrm{d}}^{0} \rightarrow \mathrm{J} / \psi \mathrm{K}_{\mathrm{S}}$ & $100 \mathrm{k}$ & $\sin (2 \beta)$ & 0.021 \\
\hline $\mathrm{B}_{\mathrm{d}}^{0} \rightarrow \pi^{+} \pi^{-}$ & 5000 & $\sin (2 \alpha)$ & 0.05 (No Penguins) \\
\hline $\mathrm{B}_{\mathrm{d}}^{0} \rightarrow \rho \pi$ & 1300 & $\alpha$ & $\sim 5^{\circ}\left(\alpha=50^{\circ}\right)$ \\
\hline $\mathrm{B}_{\mathrm{d}} \rightarrow \mathrm{D}^{* \overline{+}} \pi^{ \pm}$ & $\begin{array}{l}84 \mathrm{k} \mathrm{excl} \text {. } \\
260 \mathrm{k} \text { incl. }\end{array}$ & $2 \beta+\gamma$ & $\sim 12^{\circ}\left(2 \beta+\gamma=0, \Delta_{\mathrm{S}}=0\right)$ \\
\hline $\mathrm{B}_{\mathrm{S}}^{0} \rightarrow \mathrm{D}_{\mathrm{s}}{ }^{\mp} \mathrm{K}^{ \pm}$ & 2400 & $-2 \delta \gamma+\gamma$ & $8^{0}-12^{\circ}\left(\Delta m_{s}=15-45 p s^{-1}\right)$ \\
\hline $\mathrm{B}_{\mathrm{s}}^{0} \rightarrow \mathrm{J} / \psi \phi$ & $50 \mathrm{k}$ & $\sin (2 \delta \gamma)$ & $0.03\left(\Delta \mathrm{m}_{\mathrm{s}}=25 \mathrm{ps}^{-1}\right)$ \\
\hline
\end{tabular}




\section{CONCLUSIONS}

D LHCb is rapidly evolving towards final design since TP.

Major technology choices made.

Magnet TDR approved, RICH and Calorymeter TDR submitted.

Other Subsystem coming soon.

D Looking forward for "Ferrara 2005" for first $\angle H C b$ results on new measurements of all UT angles with unprecedented precision and may be hints for new Physics beyond the Standard Model 